\title{
Business Process Re-Engineering: Phases and Change Methodologies
}

\author{
Mustafa Shiraz Ahmed \& Naeem Janjua \\ SZABIST \\ Karachi, Pakistan
}

\begin{abstract}
Business Process Re-engineering (BPR) does not merely represent change - it means dramatic change. BPR necessitates a complete change in organizational structures, management systems, employee responsibilities and performance measurements, incentive systems, skill development, and the use of Information Technology (IT). It affects every single aspect of doing business. At one level, BPR can be regarded as the difference between complete failure and enviable success.
\end{abstract}

The following discussion includes the methods commonly employed by BPR experts and optional activities proposed by management consulting firms. BPR methods, procedures, and tasks are being identified to assist those organizations that are determined to face the unique challenges facing their respective industry, people and culture.

\section{INTRODUCTION}

Much has been written on the BPR by the practitioner trade press and the academic research journals. However, there is still no unanimity on any accurate description of BPR. Is BPR just a fad? Does it really mean a tag on to whatever your company is doing to suggest that your latest and greatest work is 'in vogue'?

Davenport \& Short [1] define Business Process (BP) as "a set of logically related tasks performed to achieve a defined business outcome. BP is a structured, measured set of activities designed to produce a specified output for a particular customer or market. It emphasizes on how work is done within an organization" [2]. In their view a BP has two important characteristics: (i) It has customers (internal or external), (ii) It has cross-organizational boundaries, i.e., it occurs across or between organizational sub units. Value Chain Method is a technique employed to identify BP in an organization.

BP is generally identified in terms of beginning and end points, interfaces, and organization units (particularly the customer unit). High impact processes should have process owners. Examples of a BP include: developing a new product; ordering goods from a supplier; creating marketing plan; processing and paying insurance claims etc.
The definition of a BP is based on three variants [1]:

- Entities: Processes take place between organizational entities - inter-organizational, inter-functional or inter -personal.

- Objects: Processes result in manipulation of objects. These objects could be physical or informational.

- Activities: Processes could involve two types of activities: Managerial (e.g. developing a budget) and Operational (e.g. filling a customer order).

BPR is the analysis and design of work flows and processes within and between organizations. Hence BPR [3] is defined as the critical analysis and radical redesign of existing business processes to achieve breakthrough improvements in performance measures.

\section{PHASES IN PERFORMING BUSINESS PROCESS RE-ENGINEERING}

Successful BPR can enormously decrease cost or cycle time. It can create substantial improvements in quality, customer service and other business objectives. Reengineering can help an aggressive company stay on top or transform an organization, which is on the verge of bankruptcy, into an effective competitor.

The success story of BPR has spawned international interest and it is now being replicated around the world. Table 1 shows the Project Phases required for successful BPR.

\begin{tabular}{|l|l|}
\hline PHASE & DESCRIPTION \\
\hline & \\
\hline 1 & Begin Organizational Change \\
\hline 2 & Build Re-engineering Organization \\
\hline 3 & Identify BPR Opportunities \\
\hline 4 & Understand the Existing Process \\
\hline 5 & Re-engineer the Process \\
\hline 6 & Blueprint the New Business System \\
\hline 7 & Perform the Transformation \\
\hline
\end{tabular}

Table 1: Project Phases Required For Successful BPR 
Phase 1: Begin Organizational Change Activities:

\begin{tabular}{|c|}
$\begin{array}{c}\text { Assess the current state of } \\
\text { the organization }\end{array}$ \\
Explain the need for \\
change
\end{tabular}

Figure 1: Activities Performed in Phase 1

Phase 2: Build the Re -engineering Organization Activities:

\begin{tabular}{|c|}
\hline $\begin{array}{c}\text { Establish a BPR } \\
\text { organizational structure }\end{array}$ \\
$\begin{array}{c}\text { Establish the roles for } \\
\text { performing BPR }\end{array}$ \\
\hline \\
Choose the personnel who \\
will reengineer
\end{tabular}

Figure 2: Activities Performed in Phase 2

\section{Phase 3: Identify BPR Opportunities}

Activities:

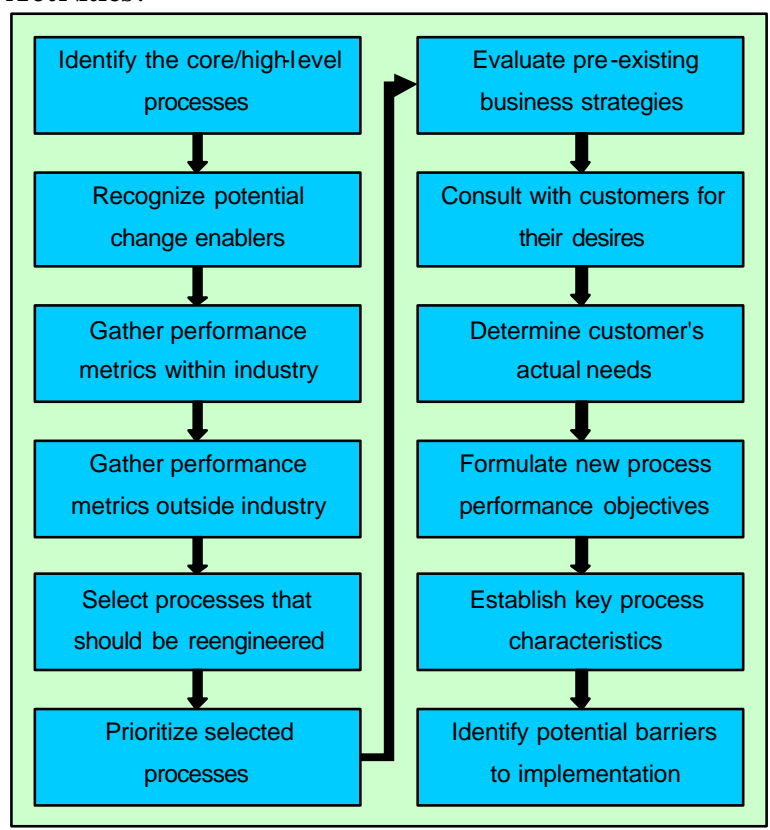

Figure 3: Activities Performed in Phase 3
Phase 4: Understand the Existing Process

Activities:

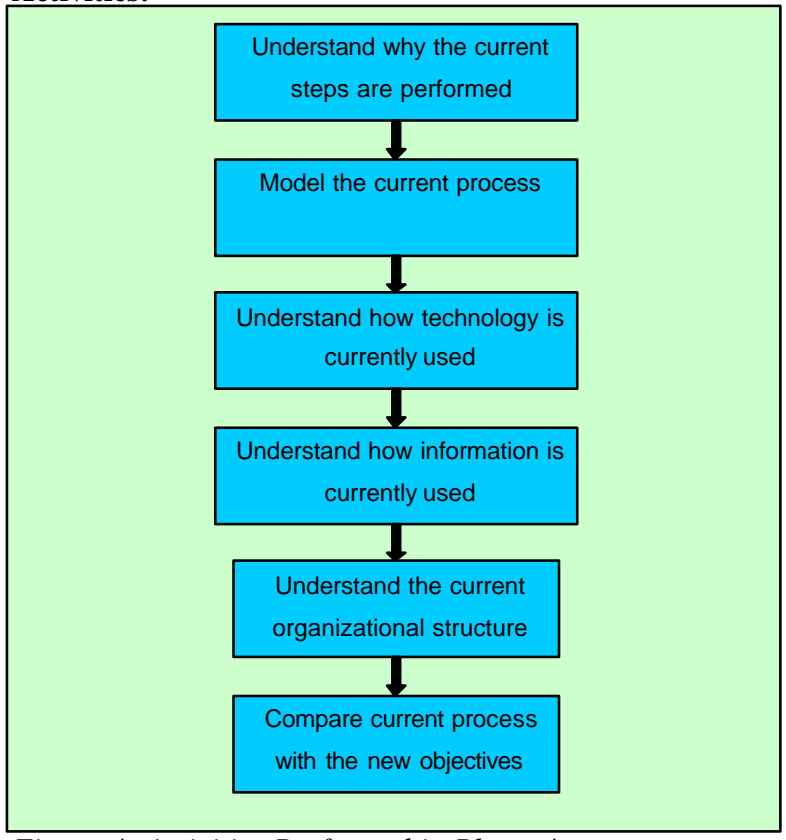

Figure 4: Activities Performed in Phase 4

Phase 5: Reengineer the Process Activities:

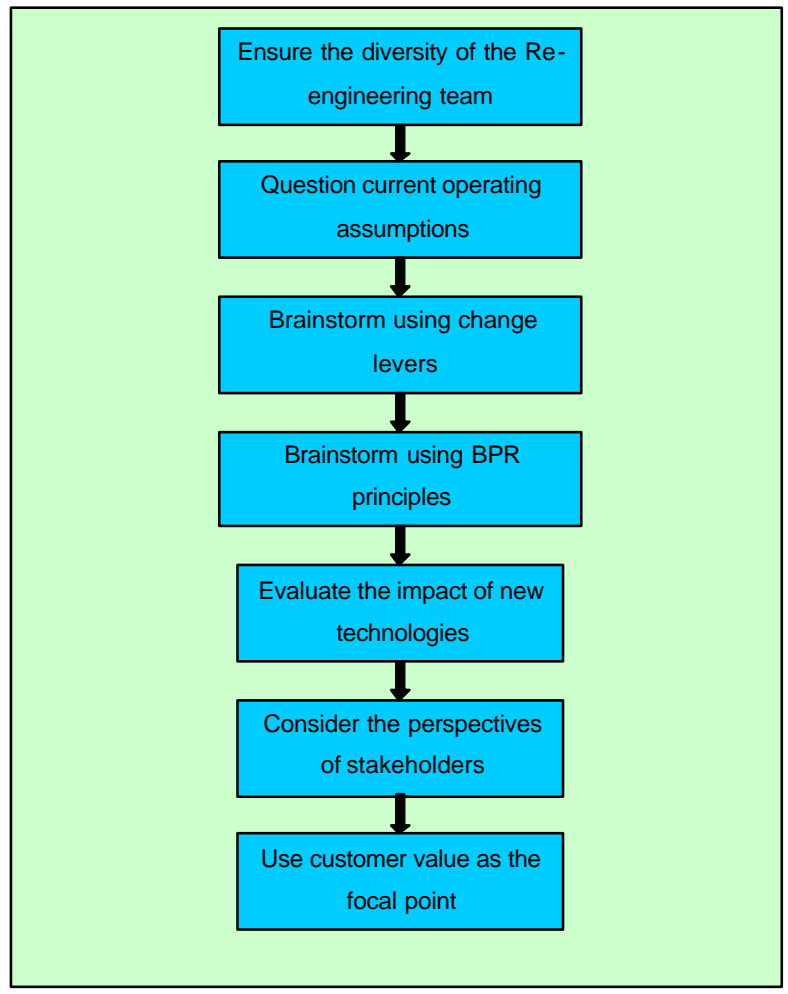

Figure 5: Activities Performed in Phase 5 
Phase 6: Blueprint the New Business System Activities:

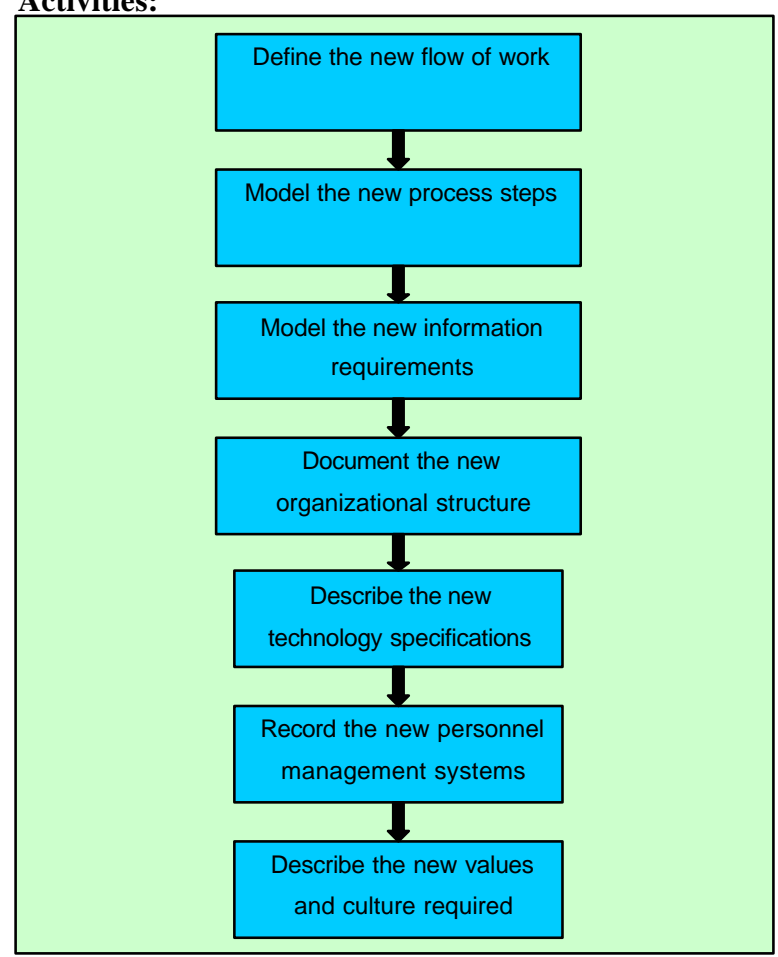

Figure 6: Activities Performed in Phase 6

Phase 7: Perform the Transformation Activities:

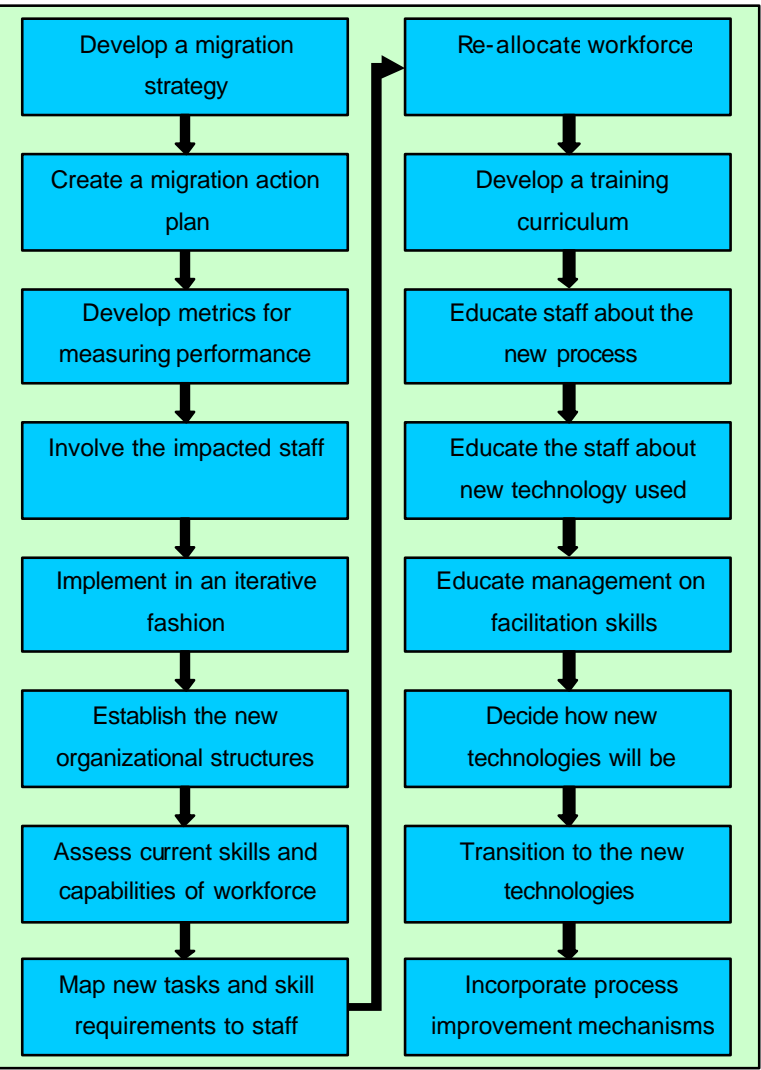

Figure 7: Activities Performed in Phase 7

\section{CHANGE METHODOLOGIES}

The success or failure of any approach depends on the ability to match the method to the desired outcome and the organizational circumstances.

It is worth noting that no particular methodology or approach is right for every organization. There are documented successes and failures for every transformational strategy. For the purpose, various change strategies have been presented by using a methodological framework, proposed by James Martin illustrated in fig. 8 .

Despite the fact that there are significant differences in the change strategies between cat egories, methodologies can be applied both one at a time or coordinating or combining methods in multiple categories.

The categories are largely differentiated by the degree of continuous or discontinuous change anticipated as a result of implementation of the change strategy.

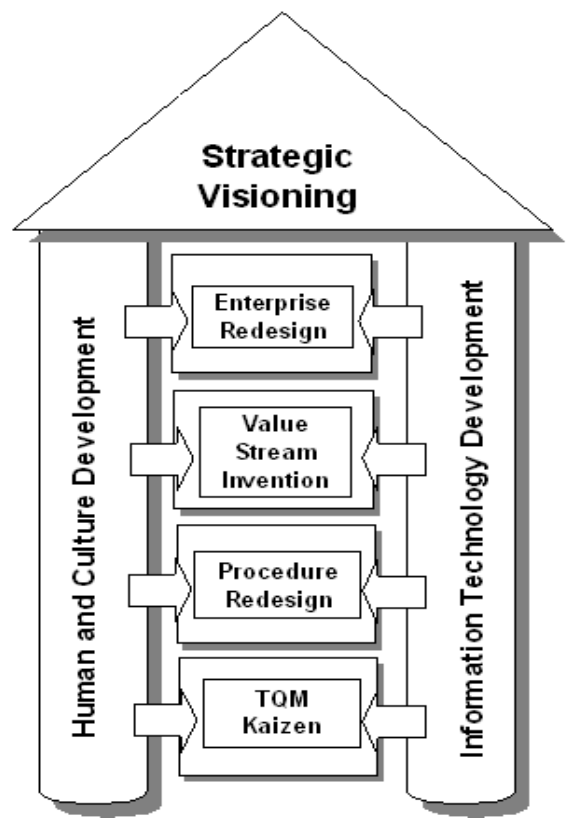

Figure 8: Strategic Visioning [4]

\section{- Continuous Process Improvement (TQM, Kaizen)}

The least invasive type of change strategy refers to the Continuous Process Improvement (CPI). CPI operates under the principle that excellence can be achieved by making a large number of small or incremental improvements continuously. The goal is to please both internal and external customers by improving the quality of both processes and outcomes.

Teamwork and individuals are encouraged and empowered to suggest and implement improvements using a structured set of tools and techniques to correctly identify and define both problems and solutions. 
This approach originated in Japan in the 1960s where it is known as "Kaizen", which means continuous improvement of products, services, customer support, relationships, systems etc. The process involves everyone within the organization.

Although there is no directly equivalent word in English, the culture of Kaizen is most closely approximated in the precepts of TQM, which gained widespread visibility in the US in the last two decades. While there is often very little agreement over how to define or measure terms like quality, there is very little disagreement with the concept that anything can be improved.

\section{- $\quad$ Procedure Re-Design}

Many organizations need more than incremental change in existing processes for the purpose of achieving the necessary results.

They don't need to make existing processes more efficient or effective; they need to identify fundamentally new ways to do business.

In many cases, taking advantage of the opportunities provided by powerful technological advances; or succeeding in increasingly competitive environments requires continuous change. But a company should not attempt to leverage technology or become more inimical without going through the fundamental process redesign. This also discourages significant investments in new technology or enterprise.

Procedure re-design is the least "invasive" of the discontinuous change strategies. It may involve streamlining of workflow, automation of activities, or improved information dissemination. It does not necessarily require replacing current processes or organizational structures.

Procedure redesign is broader in scope than TQM, often spanning multiple, cross-functional departments and/or organizations. Although it does not typically require organizational changes, it may well require installation or modification of major information systems. While broader in scope than TQM, process redesign with its emphasis on improving existing procedures is dten narrower in scope than value-stream reinvention, which focuses on replacement of existing processes. Used strategically, procedure redesign can be very effective.

\section{- Value-Stream Reinvention}

Value Stream Reinvention, like TQM, focuses on pleasing the customer. But instead of achieving this through incremental change, it necessitates radical changes in processes to achieve significant improvements. This approach substitutes a more broadly defined term "Value Stream" in lieu of "Process" to describe an "...end to end set of activities that delivers particular results to a given customer (internal or external)".

In this process the needs and desires of the customer drive the design of the process rather than customers being required to adjust to the needs of internal processes and procedures.

As the term re-invention implies, this methodology starts with a clean slate and looks for a new and the best way to structure a function or process rather than focusing on how to improve an existing one.

Every organization consists of a series of value streams. Most large organizations identify 10-20 Primary Value Streams, which represent the functions, and processes that define an organization. The first few value streams are usually designated as Strategic Value Streams. These are the areas in which the management feels they can be among the best.

Value streams that encompass functions enabling business operations are often referred as Support Value Streams. Even if those value streams have not been explicitly identified named, and managed, they exist since they correspond to how information and work actually flows in an organization.

In most of the organizations, the management structure is based on functional responsibilities rather than managing end-to-end value streams. Most of the value streams cross multiple departments or functional areas, which make the value streams slower, costlier and less satisfying to the ultimate customer.

\section{- Enterprise Re-design}

Most of the large organizations benefit from a complete redesign rather than the continual reorganization, substitution of horizontal organizational structures for vertical organizational structures, "right sizing", and other strategies commonly employed.

But the reluctance to pursue far-reaching solutions is understandable, given the inherent difficulties and increased risks associated with enterprise change.

Traditional hierarchical organizations are characterized by their ability to preserve the status quo and reject paradigm shifts and change, even when competitiveness or survival depends on them.

"As organizations grow, their cultures adapt. Mature organizations have deeply entrenched culture like the roots of an old tree. The problem with most mature corporations is that they have the wrong culture for the present era. It was set in place before the age of value streams, empowered teams, Kaizen, computer networks, and so on."

Ironically, the stronger the existing culture the more they resist change. As a result, successful organizations are 
often more at risk than new or less successful ones in periods of paradigm shifts.

Previous successes can also be a risk factor to the extent that it increases complacency and a shift in focus from external competitive factors to internal bureaucracy and maintenance of the status quo.

In the past, changes occurred so slowly that each generation of managers did not encounter obsolescence. Now technological advances recycle more quickly than management change. Hence those organizations and individuals who got the premium learn more and faster than their competitors. They also rapidly utilize the outcome of the learning process.

Now organizations must deal with how to prevent both human resource and technological obsolescence.

\section{- Strategic Visioning}

No matter how powerful an organization's technology is or how effectively it employs enterprise-engineering strategies, if it is headed in the wrong direction it won't succeed. Similarly Strategic Visioning is essential for any level of organizational transformation and functioning. As change accelerates, the need to revisit the organizational strategic assumptions and visions also increases constantly.

Unfortunately organizations often misunderstand strategic planning for Strategic Visioning. Hence it is important to understand the distinctions between these two functions. Unlike strategic planning, Strategic Visioning places a premium on intuition. It is about challenging the generally accepted assumptions and models and developing an institutional vision of what ought to be rather than what is.

It is about anticipating paradigm shifts and creating new rules where old ones no longer apply. It is about recognizing windows of opportunity to create excellence or new opportunities, products or services.

\section{REFERENCES}

[1] Davenport, T.H. \& Short, J.E. (1990 Summer) "The New Industrial Engineering: Information Technology and Business Process Redesign, "Sloan Management Review", p. 11-27.

[2] Davenport, Thomas H., Process Innovation, Boston, MA: Harvard Business School Press, 1993.

[3] Champy, J. and Hammer, M., Re-engineering the Corporation, Harper Business: New York, 1993.

[4] Martin, James, The Great Transition, Using the Seven Disciplines of Enterprise Engineering to Align People, Technology and Strategy, American Management Association, 1995. 